El futuro de la atención primaria de salud The future of Primary Health Care

\title{
ANTECEDENTES: LA REFORMA DE LA ATENCIÓN PRIMARIA
}

La reforma de la atención primaria que comenzó en el año 1985 puede considerarse concluida en el año 1998. Únicamente queda por implantarse la Zona Básica de Salud del II Ensanche de Pamplona, lo que podría ocurrir al terminar este año. La reforma ha supuesto que los recursos que se destinan a este nivel asistencial han aumentado considerablemente: el presupuesto para el año 1997 ascendía a 21.340 millones de pesetas el 33\% del gasto del Servicio Navarro de Salud; de los que 11.300 se destinaban al pago de la factura de la prestación farmacéutica.

Los cambios introducidos desde el año 1985 han supuesto una importante mejora de la asistencia. En nuestros centros de salud se presta atención a demanda y programada, en el centro y en el domicilio del paciente, incluyendo programas de prevención y promoción. Se ha generalizado el uso de la historia clinica y se trabaja por objetivos sanitarios y de gestión evaluando su cumplimiento. Algunos de los centros de salud de Navarra han elaborado programas específicos de mejora de la calidad, y todos disponen de objetivos de calidad por los que deben trabajar, objetivos que cuentan con sus correspondientes indicadores que permiten su evaluación periódica.

\section{LOS CAMBIOS QUE SE ACERCAN}

En los próximos años la atención primaria de salud debe afrontar varios cambios que afectarán directamente a su actividad, a los recursos que se le asignarán y a su forma de trabajar. Estos cambios serán consecuencia de fenómenos migratorios dentro de nuestra comunidad, cambios en la estructura

* Director de Atención Primaria y Salud Mental

Servicio Navarro de Salud 
demográfica de la población, aparición de nuevas tecnologías, mayores expectativas de los ciudadanos y cambios en la atención especializada.

La comarca de Pamplona ha sufrido importantes modificaciones en los últimos años: han surgido nuevas poblaciones como la de Mendillorri y Mendebaldea, han crecido otras como Zizur y Barañain y se despueblan el Casco Viejo y el Ensanche. Estos cambios no han terminado y se prevén en los próximos años importantes crecimientos de población en zonas como la Rochapea, San Jorge, Burlada, Milagrosa y Soto de Lezcairu.

Todos estos movimientos no responden a un crecimiento de la población de Navarra, que puede considerarse estancada, sino a cambios en los hábitos de los ciudadanos. El centro de las urbes se convierte en zona administrativa y de servicios y la población se desplaza hacia la periferia. Además la población de Navarra envejece: en 1985 los mayores de 65 años eran 69.279 (el 13,3\% del total) y en 1996 se alcanzan los 90.477 , el 17,38\% del total de la población de Navarra.

La tecnología de mayor utilización en atención primaria son los medicamentos. La industria farmacéutica se caracteriza por su continua innovación, que pone a nuestra disposición nuevos medicamentos que siempre suponen un mayor gasto. Este hecho se demuestra por el continuo crecimiento del precio medio de la receta que supera sistemáticamente el índice de precios al consumo e incluso al crecimiento del gasto sanitario.

En los próximos años se producirá una accesibilidad mayor a tecnologías diagnósticas desde la atención primaria. En unos casos porque esas tecnologías se pondrán a disposición de los médicos de familia y pediatras de atención primaria, como las endoscopias o técnicas de diagnóstico por imagen. En otros porque los médicos de atención primaria las utilizarán directamente como puede ocurrir con la ecografía, asunto éste sobre el que existe una importante controversia.

Las nuevas tecnologías de la información y de las comunicaciones están cambiando el modo de ejercer las profesiones sanitarias. En dos centros de salud de Navarra se pasa la consulta con un ordenador personal encima de la mesa, y esto es así porque la informática se ha convertido en un instrumento de mejora de la práctica clínica. Estamos cerca de la historia clínica virtual y del acceso a la información clínica de un paciente desde cualquier nivel del sistema sanitario que esté relacionado con su atención. Esta disponibilidad de información debe traducirse en una asistencia sanitaria de mejor calidad y más eficiente.

Los ciudadanos cada vez son más conscientes de sus derechos y, por lo tanto, cada vez más exigentes con el funciona- 
miento de los servicios sanitarios públicos. Además se les está informando continuamente desde los medios de comunicación, muchas veces el propio sistema sanitario público y generalmente el privado, de las excelencias de nuestros servicios o de nuevos y maravillosos tratamientos o métodos de diagnóstico. Todo ello genera demanda y mayor frecuentación de los servicios sanitarios. Todo ello genera mayores expectativas en los ciudadanos.

El sistema sanitario tiene la esperanza de que potenciando los servicios de atención primaria y su coordinación con el nivel especializado podrá mejorar la calidad conteniendo el aumento de los costes. En resumen, se pretende que la atención primaria adquiera un protagonismo cada vez mayor en la asistencia, incentivando al máximo su actividad, incluso presupuestando la mayor parte de los recursos en el nivel primario y responsabilizando a los médicos de familia de su gestión. Por ello, la atención hospitalaria está evolucionando en todos los países occidentales hacia una atención cada vez más ambulatoria. La cirugía sin ingreso o la reducción de la estancia media hospitalaria son objetivos hacia los que trabajan todos los hospitales de nuestro entorno.

Los cuidados paliativos se desplazan al nivel primario. La hospitalización a domicilio es un campo que pasará a ser del dominio de la atención primaria.

\section{LAS CONSECUENCIAS DE LOS CAMBIOS}

El envejecimiento de la población, que generará una mayor prevalencia de enfermedades crónicas, y las mayores demandas de los ciudadanos, acompañadas de unas expectativas crecientes, producirán una frecuentación mayor, tanto en consulta como en el domicilio del paciente.

La aparición de medicamentos nuevos, de mayor precio, el mayor consumo que se produce por la mayor presencia de procesos crónicos, el crecimiento del número de pensionistas, que no pagan aportación, y la mlayor frecuentación, hace suponer que el crecimiento del gasto farmacéutico será muy importante.

En resumen la demanda en atención primaria será creciente, por el envejecimiento de la población, por las mayores expectativas de los ciudadanos, por los servicios que se desplazarán desde la atención especializada y por el acceso a las tecnologías diagnósticas. El gasto de la atención primaria crecerá no sólo por la mayor demanda sino también por la incorporación de nuevas tecnologías. Además habrá que competir por los recursos con el nivel especializado, porque todos, o la mayoría de estos acontecimientos, también afectarán a la asistencia hospitalaria. 
El crecimiento de la población en determinadas zonas obligará a una redistribución de recursos que tenga en cuenta los cambios en la estructura demográfica de la población. Tendremos zonas básicas con población joven y otras con población envejecida, por lo que en unas tendremos que potenciar los servicios de pediatría y en otras la atención domiciliaria.

La atención primaria de salud debe afrontar estos cambios implicando a los profesionales en la planificación y gestión de los recursos, descentralizando la gestión en los centros de salud, adaptando los servicios a los cambios demográficos y a las nuevas expectativas de los ciudadanos, evaluando la calidad de la asistencia prestada y trabajando por mejorarla continuamente. El programa de uso racional de los medicamentos tendrá una importancia todavía mayor que en la actualidad.

La coordinación con la atención especializada será un requisito indispensable en los próximos años. La asignación de recursos a atención primaria y la imputación de los costes que se generen por la utilización de servicios hospitalarios, será el instrumento que incentivará esa coordinación, que resulta indispensable para alcanzar mayor eficiencia y efectividad del sistema sanitario.

Sin embargo, el cambio de mayor trascendencia que se producirá en los próximos años será el protagonismo creciente del médico de familia y de la estructura de atención primaria en la atención del paciente, cualquiera que sea el nivel en que esa atención se produzca, que ocasionará una profunda transformación de la organización del sistema sanitario.

Las nuevas tecnologías de la información y de las comunicaciones serán un instrumento indispensable y una indiscutible ventaja para adaptarse a los cambios que se aproximan.

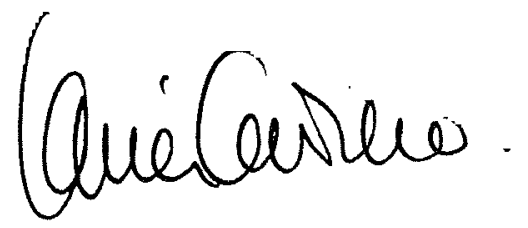

